\title{
$\begin{array}{ll}\text { Research Square } & \begin{array}{l}\text { Preprints are preliminary reports that have not undergone peer review. } \\ \text { They should not be considered conclusive, used to inform clinical practice, } \\ \text { or referenced by the media as validated information. }\end{array}\end{array}$
}

\section{Overall reduced lymphocyte subsets worsening disease severity and prognosis in COVID-19 severe cases with diabetes mellitus in Chengdu, China}

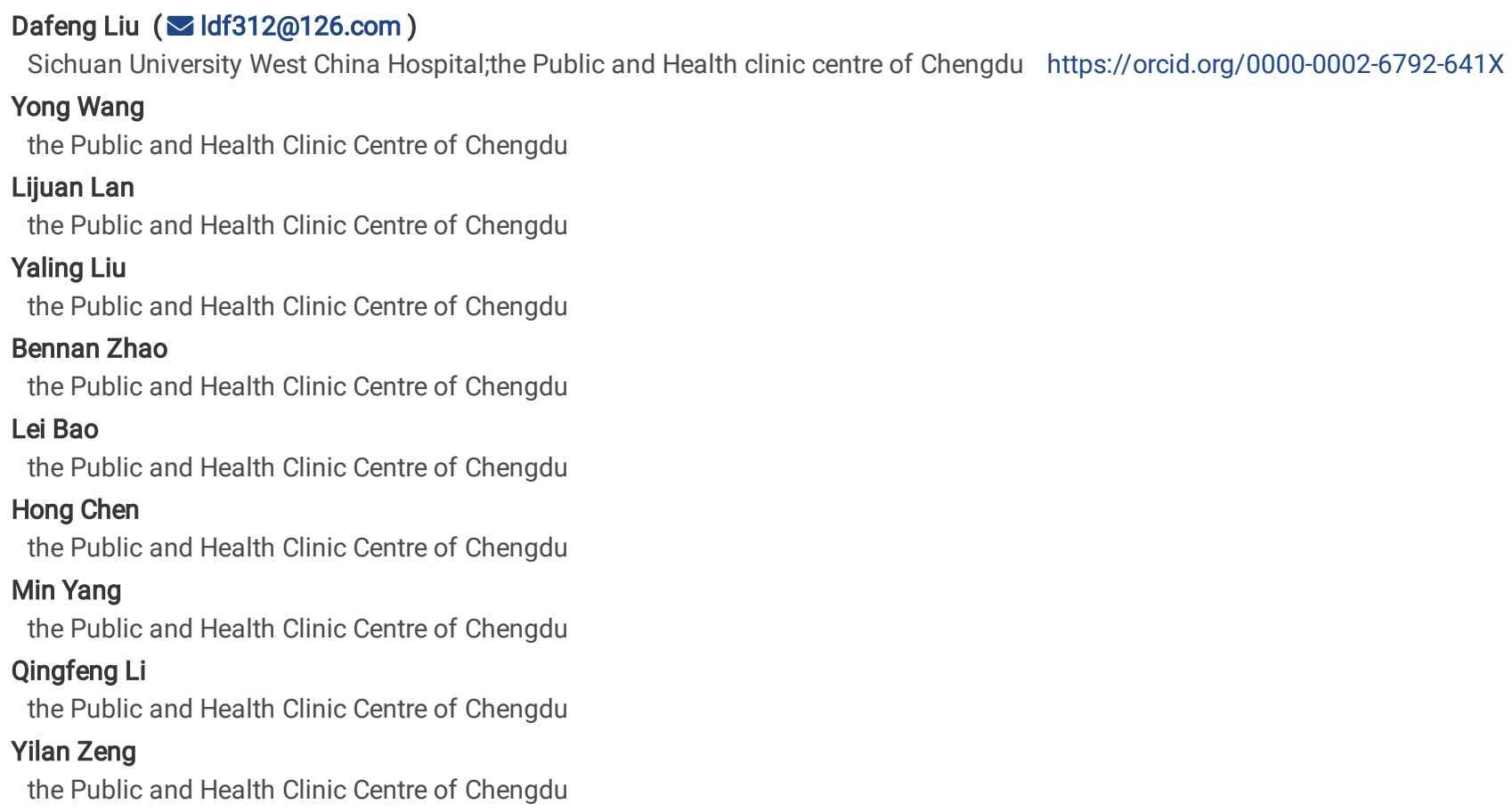

Keywords: coronavirus disease 2019 (COVID-19);lymphocyte subsets; diabetes mellitus; disease severity; viral negative conversion time; prognosis; influence factors

Posted Date: June 3rd, 2020

DOI: https://doi.org/10.21203/rs.3.rs-20385/v2

License: (c) (i) This work is licensed under a Creative Commons Attribution 4.0 International License. Read Full License 


\section{Abstract}

Background The outbreak of coronavirus disease 2019 (COVID-19) is widespread throughout China and the world.

Methods Demographic, clinical data of 95 confirmed cases with COVID-19 on admission at the Public and Health Clinic Centre of Chengdu from January 16 to March 16, 2020, were retrospectively collected and analyzed. Of them 76, 19 cases were enrolled in non-DM group (without DM), DM group (with DM), respectively; according to the disease severity 57, 19, 8, 11 cases were further divided into non-severe non-DM subgroup (light and common type and without DM), severe non-DM subgroup (severe and critical illness type and without DM), non-severe DM subgroup (light and common type and with DM), severe DM subgroup (severe and critical illness type and with DM), respectively. The severe rate and the prognosis was compared between two groups. The data of peripheral lymphocyte and subsets, age, glucose metabolism parameters were compared between four subgroups, and its relationship to the disease severity, the viral negative conversion time, and the prognosis were analyzed.

Results In this COVID-19 cohort the proportion of DM was $20 \%$. Patients with DM had significantly higher severe rate and worse prognosis than those without DM, the difference was significant (severe rate, cured, unhealed and death in DM and non-DM

groups:61.11\%vs. $25.00 \%, 26.32 \%, 68.42 \% ; 5.26 \%$ vs. $71.05 \%, 26.32 \%, 2.68 \%, x^{2}=2.940,3.394, P=0.003,0.001$,respectively), simultaneously the proportion of DM in severe cases was higher than that in non-severe cases, the obvious difference was found $\left(36.67 \%\right.$ vs.12.31\%, $\left.x^{2}=2.744, P=0.006\right)$.Severe cases with DM tended to have the lowest lymphocytes count levels and percentage values, as well as the lowest $T$ cells count levels and percentage values, helper T cells count levels and percentage values, suppressor T cells count levels, B cells count levels and percentage values compared with those severe cases without DM and non-severe cases with or without DM. The important influencing factors were that age, DM, lymphocyte percentage values and helper $T$ cells percentage values for the disease severity, lymphocyte percentage values and $B$ cell percentage values for the viral negative conversion time, and age, the disease severity and the viral negative conversion time for the prognosis.

Conclusions The COVID-19 severe cases with DM had the lowest lymphocytes count level and percentage value, especially T and B lymphocytes count levels and percentage value. Overall decreased lymphocytes subsets and DM maybe worsen prognosis by worsening the disease severity and prolonging the viral negative conversion time. Combination immunomodulatory therapy based on comprehensive treatment might improve prognosis of the COVID-19 severe cases with DM.

\section{Introduction}

Since the early of December 2019, patients with unexplained pneumonia have appeared in Wuhan, China, and they have been confirmed to be infected with a new coronavirus (2019-nCoV).[1-4]The World Health Organization (WHO) announced a new name for the epidemic disease caused by 2019-nCoV: coronavirus disease 2019(COVID-19)on 11 February 2020.COVID-19 epidemic has spread rapidly throughout the whole country, even as a world pandemic throughout globally since mid-January 2020,[5-6]as of March 26, 2020, cases were reported in China and 151 other countries, territories, and areas, a total of cumulative confirmed and death cases were 509,164 and 23,335 cases in the whole world,[5] 81394 and 3295 cases in China,[6]respectively.

Most patients with COVID-19had a good prognosis, a few patients were critically illness, it can cause respiratory, digestive, nervous and cardiovascular system damage, multiple organs failure and even death,[7-11] progress is rapid. As of Mar26, the fatality rate in China was $3.5 \sim 4.05 \%$. [6,12]The elderly and those with chronic underlying disease have a poor prognosis, diabetes mellitus(DM)is one of the common comorbiditie.[8-9,13]

Dysregulation of immune response, especially T lymphocytes, might be highly involved in the pathological process of COVID-19.[14]Increased amounts of proinflammatory cytokines in serum were associated with pulmonary inflammation and extensive lung damage in SARS,[15]MERSCOV[16]andCOVID-19.[8]Patients with DM had cellular immune dysfunction.[17]

However the characteristic of lymphocyte and subsets in patients with COVID-19 and DM coexist, the relationship of lymphocyte subsets, DM and disease progression, prognosis is unknown. In this study we aimed to analyze the difference of lymphocyte and subsets between COVID-19severe and non-severe cases with or without DM, and its influence on disease progression and prognosis.

\section{Methods}

\section{Objects}

95 patients with COVID-19 was retrospectively recruited from January 16, 2020 to March 16, 2020in hospital isolation ward of the Public and Health Clinic Centre of Chengdu, "the specific hospital for the treatment of severe patients with COVID-19 in Chengdu" designated by the government. The study was approved by the Public and Health Clinic Centre of Chengdu Ethics Committee (PJ-K2020-26-01). For emerging infectious diseases the Ethics Commission of the designated hospital agreed to waive written informed consent.

\section{The criteria of disease diagnosis, clinical typing and cured}


The disease diagnosis criteria, the clinical typing criteria, the cured criteria of COVID-19 were judged according to the seventh Trial Version of the Novel Coronavirus Pneumonia Diagnosis and Treatment Guidance.[7]

The diagnosis criteria of suspected cases were combined with the following comprehensive analysis of epidemiological history and clinical manifestations: met anyone of the history of epidemiology and any two of the clinical manifestations, and no clear epidemiological history but met three of the clinical manifestations. History of epidemiology was following: travel history or residence history of Wuhan City and surrounding areas, or other communities with case reported within 14 days before onset of illness; had a history of contact with a new coronavirus infection (positive nucleic acid test) within 14 days before onset; had contacted patients with fever or respiratory symptoms from Wuhan and surrounding areas, or from communities with case reported within 14 days before the onset of illness; aggregated disease (2 cases or more of fever and / or respiratory symptoms in small areas such as homes, offices, and school classes within 2 weeks). Clinical manifestations included following: fever and / or respiratory symptoms; with the imaging features of the above-mentioned new coronavirus pneumonia; the total number of white blood cells in the early stage of the disease was normal or decreased, or the lymphocyte count was normal or decreased.

A cross-sectional study was the research design methods.

The diagnosis criteria of confirmed cases were that suspected cases with one of the following etiological or serological evidence: real-time fluorescence RT-PCR detected the positive nucleic acid of the new coronavirus; sequencing of viral genes, highly homologous to known new coronaviruses; serum novel coronavirus-specific IgM antibodies and IgG antibodies were positive; serum novel coronavirus-specific IgG antibodies changed from negative to positive or the recovery period was 4 times or more higher than the acute period.

Clinical types included light, common, severe and critically illness four types. And typing criteria was following: The light type criteria: the clinical symptoms were mild, and there were no pneumonia manifestations on imaging; The common type criteria: the clinical symptoms included such as fever and respiratory tract, pneumonia can be seen on imaging; The severe type criteria met any of the following: Respiratory distress, RR $\geq 30$ times / $\mathrm{min}$; In the resting state, the oxygen saturation $\leq 93 \%$;Arterial blood oxygen partial pressure ( $\mathrm{PaO} 2) /$ oxygen concentration (FiO2) $\leq 300 \mathrm{mmHg}$ $(1 \mathrm{mmHg}=0.133 \mathrm{kPa})$,In areas with high altitude (over 1000 meters above sea level), $\mathrm{PaO} 2 / \mathrm{FiO} 2$ should be corrected according to the following formula: $\mathrm{PaO} 2$ / FiO2 * [atmospheric pressure ( $\mathrm{mmHg}$ ) / 760];Pulmonary imaging showed that lesions with significant progress over than $50 \%$ within 24-48 hours were managed as heavy; The critically illness type criteria included one of the following conditions: Respiratory failure occurs and mechanical ventilation is required; Shock occurs; Combining other organ failure requires ICU monitoring treatment.

The diagnostic criteria of DM were judged according to Guidelines for the Prevention and Treatment of Type 2 Diabetes in China (2017 edition).[18]

\section{The grouping and sub-grouping standards}

The participants were divided in two groups according to with and without DM: the non-DM group (patients without DM) and the DM group (patients with DM).

The participants of each group were further divided in two subgroups according to clinical typing: the non-severe non-DM subgroup (including light and common type and without DM), the severe non-DM subgroup (including severe and critically illness type and without DM), the non-severe DM subgroup (including light and common type and with DM), the severe DM subgroup (including severe and critically illness type and with DM).

\section{Definition of prognosis and the viral negative turnaround time}

The prognosis included cured, unhealed and death.

Cured discharge standard was following: the body temperature returned to normal for more than 3 days; respiratory symptoms improved significantly; lung imaging showed a significant improvement in acute exudative lesions; two consecutive sputum, nasopharyngeal swabs and other respiratory specimens tested negative for nucleic acid (sampling time at least 24 hours apart).

The viral negative conversion time was defined as the time from onset to the time of the first collection of nasopharyngeal swabs before qualifying for release of isolation.

\section{Clinical data collection}

Data, which included demographic information (age and sex), underline disease history, glucose metabolic parameters [FPG levels and hemoglobin A1c (HbA1c) levels], lymphocyte subsets, clinical data were obtained.

\section{Real-Time Reverse Transcription Polymerase Chain Reaction Assay}

Viral RNA extraction was performed using a Magnetic viral RNA/DNA extraction Kit on PAN9600 Automated Nucleic Acid Extraction System (Tianlong, Xi'an, China), according to the manufacturer's instructions, followed by polymerase-chain-reaction (PCR) screening for the presence of specific 2019-nCoV with a commercial kit (Tianlong, Xi'an, China).Saw literature.[14] 
EDTA anticoagulated peripheral blood as flow cytometry samples was drawn from the participants before initial treatment to determine lymphocyte and subsets. All samples were tested within 6 hours of being obtained. Briefly, CD3+ /CD4+ /CD8+ T-cell, CD19+ B-cell, and CD16+ CD56+ NK-cell counts (cells $/ \mu \mathrm{L}$ ) were measured by multiple-color flow cytometry with human monoclonal anti-CD3-fluorescein isothiocyanate (FITC), anti-CD4phycoerythrin (PE), antiCD8-allophycocyanin (APC), anti-CD19-PE, and anti-CD56-PE antibodies (BD Multitest) according to the manufacturer's instructions. The cells were analyzed on a BD FACS Canto II flow cytometry system (BD Biosciences) [19]

Databases were established according to the needs of the research. Two researchers simultaneously collected and entered the data. The researchers randomly selected $30 \%$ of the data for assessment to ensure data integrity, authenticity, and accuracy.

\section{Statistical analyses}

The Statistical Package for the Social Sciences software version 17.0(IBM Inc., Armonk, NY, the USA) and GraphPad Prism 8(GraphPad, CA, the USA) software were used for statistical analysis. The measurement data were expressed as $\mathrm{X} \pm \mathrm{SD}$, and a multi-group comparison was performed using ANOVA when the data had homogeneity of variance and was normally distributed, and further comparison between the two groups was conducted using least significant difference (LSD) $t$ test. Independent sample Kruskal-Wallis H(K) test was used for a multi-group comparison when the data had not homogeneity of variance, and further comparison between the two groups was conducted using Mann-Whitney $U$ test. The two groups were compared using an independent-sample $t$-test. Enumeration data was expressed in terms of percentage or proportion, and a comparison between groups was performed using Chi-square test. Spearman correlation analysis was used for two-factor correlation analysis, and multiple stepwise regression method was used for multi-factor correlation analysis. A p value of $<0.05$ was considered statistically significant.

\section{Results}

\section{Similar baseline conditions except glucose metabolic parameters between four subgroups}

95 patients with COVID-19 was retrospectively recruited, among them, 76 patients without DM were enrolled in non-DM group, including 57 nonsevere cases and 19 severe cases;19 patients with DM were enrolled in DM group, including 8 non-severe cases and 11 severe cases(of them 8 cases newly diagnosed diabetes mellitus, including 3 non-severe cases and 5 severe cases). The proportion of DM in COVID-19 was 20\%(19/95).The severe rate of patients with $\mathrm{DM}$ was higher than that of patients without $\mathrm{DM}$, the difference was significant $\left(61.11 \% v s .25 .55 \%, x^{2}=2.940, P=0.003\right)$, and the proportion of DM in severe cases was higher than that in non-severe cases, the difference was significant $\left(36.67 \%\right.$ vs. $\left.12.31 \%, x^{2}=2.744, P=0.006\right)$.

Patients in non-severe non-DM subgroup were significantly younger than those in the other three subgroups, but those of other three subgroups had similar age(Table 1). No significant difference was observed in terms of gender between four subgroups(Table 1). FPG levels and HbA1c levels in DM group were obviously higher than that in non-DM group(Table 1), and FPG levels and HbA1c levels in severe non-DM subgroup were higher than that in non-severe non-DM subgroup. But there was no significant difference of glucose metabolic parameters between non-severe DM subgroup and severe DM subgroup(Table 1).

Table 1. Comparison of baseline conditions and glucose metabolic parameters between four subgroups $n=95 \square$

\begin{tabular}{|c|c|c|c|c|c|c|c|c|c|c|}
\hline \multirow[t]{3}{*}{ variable } & \multicolumn{4}{|c|}{ non-DM group $\square n=76 \square$} & \multicolumn{4}{|c|}{ DM group $(n=19)$} & \multirow[t]{3}{*}{$\square^{2}$ or F score } & \multirow[t]{3}{*}{$P$ score } \\
\hline & \multicolumn{2}{|c|}{ non-severe subgroup $(n=57)$} & \multicolumn{2}{|c|}{ severe subgroup $(n=19)$} & \multicolumn{2}{|c|}{ non-severe $\operatorname{subgroup}(n=8)$} & \multicolumn{2}{|c|}{ severe subgroup $(n=11)$} & & \\
\hline & case & $\mathrm{x} \pm \mathrm{SD}$ & case & $\mathrm{x} \pm \mathrm{SD}$ & case & $\mathrm{x} \pm \mathrm{SD}$ & case & $\mathrm{x} \pm \mathrm{SD}$ & & \\
\hline age $\square$ year $\square$ & 57 & $42.67 \pm 14.71$ & 19 & $58.00 \pm 19.24 * * *$ & 8 & $61.57 \pm 12.01 * * *$ & 11 & $59.36 \pm 12.31 * * *$ & $F=8.958$ & 0.000 \\
\hline male $\llbracket$ case $\llbracket \% \square$ & 57 & $25(43.86)$ & 19 & 11(57.89) & 8 & $3(37.50)$ & 11 & $7(63.64)$ & $\square^{2}=2.532$ & 0.469 \\
\hline FPG $\square m m o l / L \square$ & 57 & $5.35 \pm 0.65$ & 19 & $5.81 \pm 0.91$ & 8 & $7.48 \pm 4.63 * * * * \# \#$ & 11 & 7.35 $1.19 * * * * \# \#$ & $F=8.621$ & 0.000 \\
\hline $\mathrm{HbA1c}(\%)$ & 57 & $4.77 \pm 0.54$ & 19 & $5.10 \pm 0.62$ & 8 & 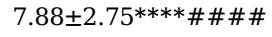 & 11 & 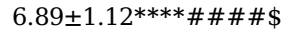 & $F=33.485$ & 0.000 \\
\hline
\end{tabular}

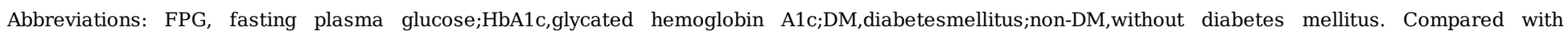

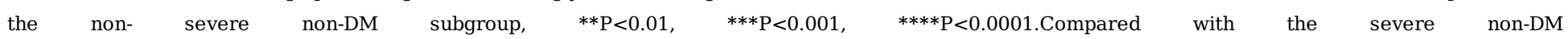

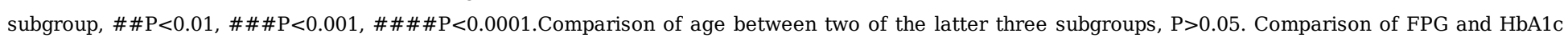
between the former two subgroups, and between the latter two subgroups, all $\mathrm{P}>0.05$.

\section{Overall decreased lymphocytes subsets in COVID-19 severe cases with DM}

In COVID-19 severe cases with DM lymphocyte count level, lymphocyte percentage value,CD3+ count level and percentage value,CD3+CD4+ count level and percentage value,CD3+CD8+ count level, B(CD19+)count level and percentage value were the lowest compared with that in the other three subgroups, especially non-severe non-DM and non-severe DM subgroups, the difference was significant (Table 2). 
Table 2. Comparison of lymphocyte and subsets at baseline between four subgroups $n=95 \square$

\begin{tabular}{|c|c|c|c|c|c|c|c|c|c|c|c|}
\hline \multirow[t]{3}{*}{ variable } & & \multicolumn{4}{|c|}{ non-DM group $\llbracket n=76 \square$} & \multicolumn{4}{|c|}{ DM group $(n=19)$} & \multirow{3}{*}{$\begin{array}{l}F \text { or } Z \\
\text { score }\end{array}$} & \multirow[t]{3}{*}{$P$ score } \\
\hline & & \multicolumn{2}{|c|}{$\begin{array}{l}\text { non- } \\
\text { severe subgroup }(n=57)\end{array}$} & \multicolumn{2}{|c|}{ severe subgroup $(n=19)$} & \multicolumn{2}{|c|}{$\begin{array}{l}\text { non- } \\
\text { severe } \operatorname{subgroup}(n=8)\end{array}$} & \multicolumn{2}{|c|}{ severe subgroup $(n=11)$} & & \\
\hline & & case & $\mathrm{x} \pm \mathrm{SD}$ & case & $\mathrm{x} \pm \mathrm{SD}$ & case & $\mathrm{x} \pm \mathrm{SD}$ & case & $\mathrm{x} \pm \mathrm{SD}$ & & \\
\hline \multirow[t]{2}{*}{ lymphocyte } & LY & 57 & $1125.07 \pm 574.68$ & 19 & $827.58 \pm 446.06$ & 8 & $1122.63 \pm 277.73$ & 11 & $538.91 \pm 324.18^{* *} \# \$ \$$ & $Z=15.049$ & 0.002 \\
\hline & LY\% & 57 & $13.26 \pm 6.30$ & 19 & $9.34 \pm 5.93 *$ & 8 & $11.99 \pm 5.64$ & 11 & $6.58 \pm 3.47^{* *} \$$ & $Z=14.661$ & 0.002 \\
\hline \multirow[t]{6}{*}{$\begin{array}{l}\text { lymphocyte } \\
\text { subsets }\end{array}$} & $\begin{array}{l}\text { CD3+ } \\
\text { (cells/ul) }\end{array}$ & 57 & $837.51 \pm 471.89$ & 19 & $538.00 \pm 314.10^{* *}$ & 8 & $830.00 \pm 194.74$ & 11 & $395.36 \pm 285.31 * * \$$ & $F=5.283$ & 0.002 \\
\hline & $\mathrm{CD} 3+\%$ & 57 & $73.61 \pm 8.18$ & 19 & $74.48 \pm 6.62 * * * *$ & 8 & $68.29 \pm 9.76 \#$ & 11 & $58.54 \pm 10.70 \# \# \$$ & $Z=16.567$ & 0.001 \\
\hline & $\mathrm{B}(\mathrm{CD} 19+)$ & 57 & $121.32 \pm 71.85$ & 19 & $122.74 \pm 84.42$ & 8 & $132.50 \pm 84.04$ & 11 & $51.09 \pm 34.96 * \# \# \$ \$$ & $Z=12.916$ & 0.005 \\
\hline & $\mathrm{B}(\mathrm{CD} 19+) \%$ & 57 & $10.59 \pm 4.39$ & 19 & $14.90 \pm 7.05^{* *}$ & 8 & $11.33 \pm 4.79$ & 11 & $8.86 \pm 4.82 \# \#$ & $F=4.361$ & 0.006 \\
\hline & NK(CD56+) & 57 & $154.98 \pm 105.91$ & 19 & $138.53 \pm 106.33$ & 8 & $138.25 \pm 82.43$ & 11 & $91.00 \pm 58.79$ & $Z=5.469$ & 0.140 \\
\hline & NK(CD56+)\% & 57 & $13.61 \pm 7.02$ & 19 & $17.19 \pm 9.99$ & 8 & $13.73 \pm 9.48$ & 11 & $14.90 \pm 7.16$ & $Z=2.378$ & 0.426 \\
\hline \multirow{5}{*}{$\begin{array}{l}\mathrm{T} \quad \text { cell } \\
\text { subsets }\end{array}$} & CD3+CD4+ & 57 & $484.72 \pm 285.85$ & 19 & $280.21 \pm 157.20^{* *}$ & 8 & $523.75 \pm 142.24 \#$ & 11 & $233.27 \pm 181.96 * * \$$ & $F=5.960$ & 0.001 \\
\hline & $\mathrm{CD} 3+\mathrm{CD} 4+\%$ & 57 & $42.38 \pm 8.96$ & 19 & $33.71 \pm 9.49^{* * *}$ & 8 & $46.73 \pm 6.42 \# \#$ & 11 & $41.10 \pm 12.36^{* *} \#$ & $Z=13.775$ & 0.003 \\
\hline & CD3+CD8+ & 57 & $289.61 \pm 175.00$ & 19 & $223.63 \pm 178.27 *$ & 8 & $253.00 \pm 90.00$ & 11 & $141.36 \pm 105.35^{* *} \$$ & $Z=14.051$ & 0.003 \\
\hline & CD3+CD8+\% & 57 & $25.80 \pm 6.59$ & 19 & $26.00 \pm 10.57$ & 8 & $22.33 \pm 7.26$ & 11 & $27.77 \pm 10.94$ & $F=0.708$ & 0.550 \\
\hline & CD4/CD8 & 57 & $1.82 \pm 0.80$ & 19 & $1.60 \pm 0.86$ & 8 & $2.49 \pm 1.15$ & 11 & $1.83 \pm 1.18$ & $Z=3.992$ & 0.262 \\
\hline
\end{tabular}

Abbreviations: DM, diabetes mellitus; LY, lymphocyte. Compared with the non- severe non-DM

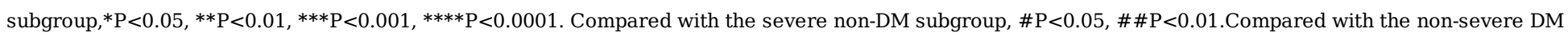
subgroup, $\$ \mathrm{P}<0.05, \$ \mathrm{P}<0.01$. Comparison of other parameters between other two subgroups, all $\mathrm{P}>0.05$.

In COVID-19 severe cases without DM, lymphocyte percentage value,CD3+ count level and percentage value, CD3+CD4+ count level and percentage value, $C D 3+C D 8+$ count level and $B(C D 19+)$ percentage value were lower than that in non-sever patients without DM, there were obviously statistical difference(Table 2).

In COVID-19 severe cases with DM, lymphocyte count level and percentage value,CD3+ count level and percentage value, CD3+CD4+ count level, CD3+CD8+ count level and B(CD19+)count level were lower than that in non-sever patients with DM, obviously statistical differences were also found (Table 2).

\section{More severe rate, longer virus negative conversion time and worse prognosis in COVID-19 severe cases with DM}

In COVID-19 patients with DM the severe rate was higher and the prognosis was worse, moreover in COVID-19 patients with DM whether severe cases or not, the virus negative conversion time were longer than that in those without DM, patients in severe DM subgroup had the longest in-hospital time compared with that in the other three subgroups, the differences were significant (Table 3 ).

Table 3. Comparison of the disease severity, the virus negative conversion time and the prognosis between four subgroups $\square \mathrm{n}=95 \square$

\begin{tabular}{|c|c|c|c|c|c|c|}
\hline \multirow[t]{2}{*}{ iriable } & \multicolumn{2}{|l|}{ non-DM group $\llbracket n=76 \square$} & \multicolumn{2}{|l|}{ DM group $(n=19)$} & \multirow{2}{*}{$\begin{array}{l}\square^{2} \text { or } F \text { or } Z \\
\text { score }\end{array}$} & \multirow[t]{2}{*}{$P$ score } \\
\hline & $\begin{array}{l}\text { non- } \\
\text { severe subgroup }(n=57)\end{array}$ & severe subgroup $(n=19)$ & $\begin{array}{l}\text { non- } \\
\text { severe } \operatorname{subgroup}(n=8)\end{array}$ & severe subgroup $(n=11)$ & & \\
\hline $\begin{array}{l}\text { rus negative conversion } \\
\text { ne }\end{array}$ & $18.49 \pm 10.02$ & $20.53 \pm 9.25$ & $28.00 \pm 12.84^{*}$ & $27.73 \pm 9.57 * *$ & $Z=11.905$ & 0.008 \\
\hline hospital time & $14.25 \pm 8.72$ & $19.79 \pm 12.33$ & $19.38 \pm 8.12$ & $29.27 \pm 16.59 * * * * \# \$$ & $F=6.704$ & 0.000 \\
\hline ’vere $\llbracket$ case $\llbracket$ $\%$ & 19(25.00) & & $11(61.11)$ & & $\square^{2}=-2.940$ & 0.003 \\
\hline ognosis & & & & & $\square^{2}=-3.394$ & 0.001 \\
\hline cured(case $\square \% \square$ & $53 \square 71.05 \square$ & & $5(26.32)$ & & & \\
\hline unhealed & $21(26.32)$ & & $13(68.42)$ & & & \\
\hline death & $2(2.63)$ & & $1(5.26)$ & & & \\
\hline
\end{tabular}

Abbreviations: FPG, fasting plasma glucose;HbA1c,glycated hemoglobin A1c;DM,diabetesmellitus;non-DM, without diabetes mellitus. Compared with the nonsevere non-DM subgroup, ${ }^{*} \mathrm{P}<0.05, * * \mathrm{P}<0.01, * * * * \mathrm{P}<0.0001$. Compared with the severe non-DM subgroup, \# $\mathrm{P}<0.05$.Compared with the non-severe $\mathrm{DM}$ subgroup, $\$ \mathrm{P}<0.05$.Comparison of the virus negative conversion time and the inhospital time between two other subgroups, all $\mathrm{P}>0.05$. 
Spearman correlation analysis showed that DM and age were positively, while lymphocyte count level, lymphocyte percentage value,CD3+ count level, CD3+CD4+ count level, CD3+CD8+ count level, CD3+ percentage value, CD3+CD4+ percentage value, B (CD19+) count level were negatively related to the disease severity (Table 4). The important factors influencing the disease severity by multiple stepwise regression analysis was DM, age, lymphocyte percentage value and CD3+CD4+ percentage value (Table 5).

Only DM and age were positively related to the viral negative conversion time (Table 4) by Spearman correlation analysis. Influencing factors of the virus negative conversion time by multiple stepwise regression analysis was lymphocyte percentage value and B (CD19+) percentage value (Table $5)$.

$\mathrm{DM}$, age, the disease severity and the coronavirus negative conversion time were positively related to the prognosis, while lymphocyte percentage value was negatively related to the prognosis by Spearman correlation analysis (Table 4). Influencing factors of the prognosis by multiple stepwise regression analysis was age, the disease severity and the coronavirus negative conversion time (Table 5).

Table 4.Spearman correlation analysis of the disease severity,virus negative conversion time,prognosis,lymphocyte subsets, age and $\mathrm{DM} \square n=95 \square$

\begin{tabular}{|c|c|c|c|c|c|c|}
\hline \multirow[t]{2}{*}{ variable } & \multicolumn{2}{|c|}{$\begin{array}{lr}\text { disease } & \text { severity }(1= \\
\text { severe }, 3=\text { critically illness })\end{array}$} & \multicolumn{2}{|c|}{$\begin{array}{c}\text { common, } 2=\text { virus } \text { negative } \mathrm{c} \\
\text { time(days) }\end{array}$} & \multicolumn{2}{|c|}{ conversionprognosis $(1=$ cure, $2=$ unheal, $3=$ death $)$} \\
\hline & $\bar{r}$ & $\mathrm{p}$ & $\mathrm{r}$ & $\mathrm{p}$ & $\mathrm{r}$ & $\mathrm{p}$ \\
\hline$\overline{\mathrm{DM}}(1=$ =without,2=with $)$ & 0.320 & 0.000 & 0.337 & 0.001 & & \\
\hline age(year) & 0.361 & 0.000 & 0.264 & 0.010 & 0.263 & 0.010 \\
\hline LY (cells/ul) & -0.341 & 0.001 & & & & \\
\hline LY\%(\%) & -0.371 & 0.000 & & & -0.209 & 0.042 \\
\hline CD3+(cells/ul $)$ & -0.379 & 0.000 & & & & \\
\hline CD3+CD4+(cells/ul) & -0.388 & 0.000 & & & & \\
\hline CD3+CD8+(cells/ul) & -0.351 & 0.000 & & & & \\
\hline $\mathrm{CD}+3 \%(\%)$ & -0.302 & 0.003 & & & & \\
\hline $\mathrm{CD} 3+\mathrm{CD} 4+\%$ & -0.219 & 0.033 & & & & \\
\hline CD19+(cells/ul) & -0.266 & 0.033 & & & & \\
\hline $\begin{array}{l}\text { disease } \quad \text { severity }(1=\text { common }, 2= \\
\text { severe }, 3=\text { critically illness })\end{array}$ & & & & & 0.331 & 0.001 \\
\hline virus negative conversion time(days) & & & & & 0.299 & 0.003 \\
\hline
\end{tabular}

Abbreviations: DM, diabetesmellitus. LY, lymphocytes.

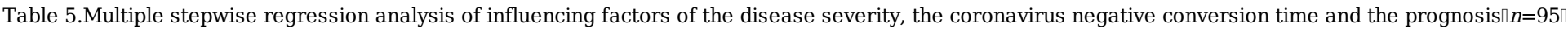

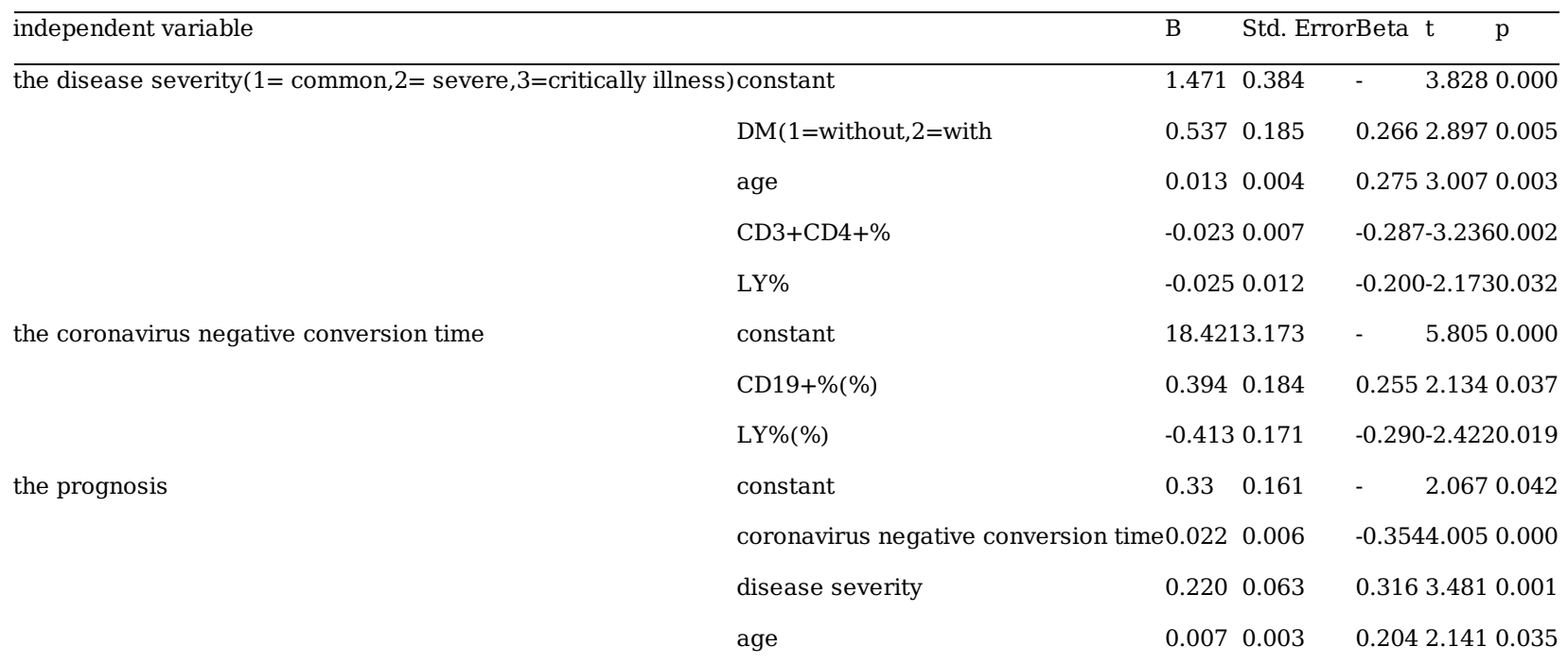

Abbreviations: DM, diabetes mellitus. LY, lymphocytes count. 


\section{Discussion}

DM is one of the common comorbidities in COVID-19,[8-9,20]the proportion of DM in this COVID-19 cohort was 20\%(19/95),consistent with those reports $20 \%$ (8/41),[8]higher than those reports $8 \%$ (3700/46248)[21]and 13\% (13/99),[9] the proportion of DM in severe cases of this COVID-19 cohort was $36.67 \%(11 / 30)$,significantly higher than $12.31 \%(8 / 65)$ in non-severe cases. These finds were not consistent with those reports that there is no significant difference of the prevalence of DM between non-severe cases and severe cases.[14]The reasons for the inconsistent findings may be that 8 cases of DM in this COVID-19 cohort were newly diagnosed after admission, of which 3 cases in the non-severe group and 5 cases in the severe group. Therefore, for patients with COVID-19 close monitoring of blood glucose, detection of glycated hemoglobin and performing of glucose tolerance test are necessary to find potential DM in time. Further using correlation analysis we found that DM was positively related to the disease severity and the viral negative conversion time, by multiple stepwise regression analysis we found that DM was an important influencing factor of the disease severity, this was consistent with previous research finds that DM was closely related to the disease severity and death of patients with viral pneumonia. For example, in 2003 , for acute respiratory distress syndrome (SARS) patients with DM, the rate of mortality, check in the intensive care units, and the need for mechanical ventilation were from 3.0 to 3.3 times that of those without DM,[22]the rate of check in the intensive care units for H1N1 influenza patients with DM was 4.29 times that of those without DM,[23]DM was also a high-risk factor for the Middle East Respiratory Syndrome Coronavirus infection severe cases in 2014.[23]

In this study we found that COVID-19 patients with DM had the highest severe rate, COVID-19 severe cases with DM had the worst prognosis, and overall the lowest lymphocyte and subsets especially CD4+, CD8+ T and B cells, consistent with those reports.[14]But hierarchical analysis was not performed based on COVID-19 patients with or without DM in the literature.[14]

Further using correlation analysis we found that lymphocyte and subsets were negatively related to the disease severity, lymphocyte was negatively related to the prognosis. By multiple stepwise regression analysis we found that important influencing factors of the disease severity included DM, age, CD4+ percentage and lymphocyte percentage, of the prognosis involved the virus negative conversion time, the disease severity and age.

We also found that COVID-19 severe cases with DM had the lowest B (CD19+) cell count levels and the longest virus negative conversion time. By multiple stepwise regression analysis we found that important influencing factors of the virus negative conversion time consist of lymphocyte percentage value and B (CD19+) percentage value. Those discovery was consistent with those reports[14] that there was no significant difference of $\mathrm{B}(\mathrm{CD} 19+)$ count levels or percentage values between COVID-19 non-severe cases and severe cases.

In this study we found that non-severe patients without DM were significantly younger than severe patients without DM, consistent with those reports that those COVID-19 patients who is the elderly have a poor prognosis.[8-9,13]Previous study found that the landscape of CD4 T cell subsets differs markedly between young and old mice, such that three cell subsets-exhausted, cytotoxic, and activated regulatory T cells (a Tregs)-appear rarely in young mice but gradually accumulate with age. Most unexpected were the extreme pro- and anti-inflammatory phenotypes of cytotoxic CD4 T cells and a Tregs, respectively.[24]Total numbers and relative frequencies of B cells were found to decline upon aging, with reductions in transitional B cells, memory cell types, and plasma blasts in the $70+$ y group.[25]Lymphocytes and subsets including T(CD3+) cell, B(CD19+) cell and NK(CD56+) cell are mainly responsible for host immune regulation, $\mathrm{T}$ cells play a dominant role in promoting or sustaining inflammatory processes through inducing proinflammatory cytokines production.[23,26-29]Activated Th1 cells, one subtype of CD4+ effector T cell, could trigger phagocytedependent inflammation and cell mediated immunity by producing tumor necrosis factor-beta(TNF- $\beta$ ), interleukin-2 (IL-2), interferon- gamma(IFN- $\gamma$ ). [23]In contrast, activated Th2 cells, another subtype of CD4+ effector T cell, can regulate antibody responses by produce IL-4, IL-5, IL-6, IL-9, IL-10, and IL-13.[26]Virus infection through inducing direct cytopathic effects and indirect host immune responses played major roles in disease progression.[14,27]The first line of defense against viral invisions was a rapid and well-coordinated innate host immune response, but dysregulated immune response will result in an excessive inflammation, even cause death.[14]

Severe patients without DM were older than non-severe patients without DM. Furtherusing Spearman correlation analysis we found that age was positively related to the disease severity, the viral negative conversion time and the prognosis, by multiple stepwise regression analysis we found that age was an important influencing factor of the disease severity and the prognosis, this was consistent with those reports that the elderly have a poor prognosis.[8-9,13] However non-severe patients with DM and severe patients with DM had similar age, this means that age is not a factor for severity in patients with DM, unconsistent with those reports.[8-9,13]Type 2 diabetes mellitus( T2DM) is a systemic chronic low-grade inflammatory disease. An altered function of specific T lymphocyte populations, including $T$ regulatory (Treg) cells, which led to the hypothesis that part of the inflammatory response mounting in T2D is attributable to an autoimmune phenomenon.[30]T cells play a dominant role in promoting or sustaining inflammatory processes and insulin resistance through inducing pro-inflammatory cytokines in metabolic organs, such as the adipose tissue, liver, muscle, and pancreas.[29,31-33]Macrophages are the major inflammatory cells in the adipose tissue.[29,31,33]Proinflammatory M1 macrophages contribute to the local and systemic inflammation by releasing proinflammatory cytokines such as TNF-a, IL-6, and IL-1.[33]On the contrary, antiinflammatory M2 macrophages inhibit the activity of most pro-inflammatory cell types including M1 macrophages by secreting IL-10.[33]IL-10 has been shown to suppress TNF-a by interacting with the p38/MAPK pathway.[33]Th1 cells promote M1 polarization and enhance its pro-inflammatory functions by producing TNF-a,IL-2,IFN-Y. In contrast, Th2 cells skew the differentiation of macrophage towards M2 by producing anti-inflammatory IL-4,and IL-13.[29,31-33]Therefore, Th1 and Th2 responses, which are closely related to M1/M2 polarization, may also have a critical role in T2DM.

$[29,31-33]$ 
Previous research found that in COVID-19 patients higher expression of proinflammatory cytokines and chemokines, especially in the severe cases, the consumption of CD4+ and CD8+ T cells, and the decrease of regulatory T cells, might result in aggravated inflammatory responses, the production of cytokine storm and make damaged tissue worse. Maybe lower number of lymphocytes suggested a role for dysregulated immune responses in COVID-19 pathogenesis.[14]Our research discoveries suggested that the coexistence of viral infection and DM result in more dysregulated host immune responses thus worsen the already aggravated inflammatory process, more susceptible to bacterial infections, more severe organ damage and worse prognosis, simultaneously the coexistence of viral infection and DM can reduce or delay antibody production by decreasing $\mathrm{B}(\mathrm{CD} 19+)$ count level and percentage value, thereby delaying the removal of the virus, worsening prognosis.

Our study had several limitations. First, it was a retrospective, single center and small sample study. Second, CD4+ effector T cells subtypes such as pro-inflammatory Th1, Th17, and anti-inflammatory Th2 and Foxp3+ regulatory T cell (Treg),and macrophages two populations such as proinflammatory M1 and anti-inflammatory M2 were not further identified. Despite that, our study demonstrated several novel information that the coexistence of viral infection and DM result in more dysregulated host immune responses thus worsen the already aggravated inflammatory process, more susceptible to bacterial infections, more severe organ damage and worse prognosis, simultaneously the coexistence of viral infection and DM can reduce or delay antibody production, thereby delaying the removal of the virus, worsen prognosis.

\section{Conclusions}

The COVID-19 severe cases with DM had the lowest lymphocytes, especially T and B lymphocytes. Overall decreased lymphocytes subsets and DM maybe aggravated the prognosis by aggravating the disease severity and prolonging the viral negative conversion time. Combination immunomodulatory therapy based on comprehensive treatment might improve prognosis of the COVID-19 severe cases with DM.

\section{Declarations}

\section{ACKNOWLEDGMENTS}

Thanks to Dr. Ling Zhang, Mingquan Zeng (the Public and Health Clinic Centre of Chengdu, one isolation ward, Clinical Medical Laboratory, respectively).

\section{CONFLICT OF INTERESTS}

The authors declare that they have no competing interests.

\section{FUNDING}

This research was supported by the Sichuan Province Health Commission (17PJ070), Chengdu Municipal Health Commission(2019079),Chengdu Science and Technology Bureau(2020-YF05-00191-SN).

\section{AUTHORS' CONTRIBUTIONS}

Concept and design: Dafeng Liu,Yong Wang, Lijuan Lan, Yaling Liu, Bennan Zhao; Data acquisition: Dafeng Liu,Yong Wang, Lijuan Lan, Yaling Liu, Bennan Zhao, Lei Bao; data analysis and interpretation: Dafeng Liu,Yong Wang, Lijuan Lan, Yaling Liu, Bennan Zhao; Drafting the manuscript: Dafeng Liu,Yong Wang, Lijuan Lan, Yaling Liu, Bennan Zhao; administrative, technical, or material support:Dafeng Liu,Yong Wang, Lijuan Lan, Yaling Liu, Bennan Zhao, Lei Bao,Hong Chen,Min Yang, Qingfeng Li;study supervision: Yilan Zeng.

\section{ORCID}

Dafeng LiuiDhttps://orcid.org/0000-0002-6792-641X

\section{DATA AVAILABILITY STATEMENT}

All data, models, or code generated or used during the study are available from the corresponding author by request: Dafeng Liu,Email:liudf312@126.com

\section{References}

1. Wu G, Gao GF, Tan W, et al. A Novel Coronavirus from Patients with Pneumonia in China, 2019. N Engl J Med. 2020; 382(8):727-733.

2. Lu H, Stratton CW,Tang YW. Outbreak of Pneumonia of Unknown Etiology in Wuhan China: the Mystery and the Miracle. J Med Virol. 2020; 92(4):401-402.

3. Ji W, Wang W, Zhao X, et al. Cross-species transmission of the newly identified coronavirus 2019-nCoV.J Med Virol. 2020; 92(4):433-440.

4. Gates B. Responding to COVID-19 - A Once-in-a-Century Pandemic? N Engl J Med. 2020 Feb 28. doi: 10.1056/NEJMp2003762. Online ahead of print. 
5. World Health Organization. Coronavirus disease 2019 (COVID-19) situation report-67, 27 Mar 2020 [cited 2020 Mar27]. Available at: https://www.who.int/docs/default-source/coronaviruse/situation-reports/20200327-sitrep-67-covid-19.pdf[External Link]

6. National Health Commission of the People's Republic of China. Update on the epidemic situation of new coronavirus pneumonia as of 24:00 on March 27,2020. Available at: http://www.nhc.gov.cn/xcs/yqfkdt/202003/bf66696029ba420098 shtm[External Link]

7. National Health Commission of the People's Republic of China. The seventh Trial Version of the Novel Coronavirus Pneumonia Diagnosis and Treatment Guidance. Available at:http://medjournals.cn/2019NCP/index.do;jsessionid=F12 B0B0FEBD6E6193A01B01FEA4E8109][External Link]

8. Huang C, Wang Y, Li X, et al. Clinical features of patients infected with 2019 novel coronavirus in Wuhan, China. 2020; 395(10223):497-506.

9. Chen N, Zhou M, Dong X, et al. Epidemiological and clinical characteristics of 99 cases of 2019 novel coronavirus pneumonia in Wuhan, China: a descriptive study. Lancet.2020; 395(10223):507-513.

10. Wang D, Hu B, Hu C, et al., Clinical characteristics of 138 hospitalized patients with 2019 novel coronavirus-infected pneumonia in Wuhan, China. 2020 Feb 7:e201585. doi: 10.1001/jama.2020.1585. Online ahead of print.

11. Guan WJ, Ni ZY, Hu Y, et al. Clinical Characteristics of 2019 Novel Coronavirus Infection in China. medRxiv preprint doi: https://doi.org/10.1101/02.06. 20020974.

12. Wilson N, Kvalsvig A, Barnard LT,et al. Case-Fatality Risk Estimates for COVID-19 Calculated by Using a Lag Time for Fatality. Emerg Infect Dis. 2020 Mar 13; 26(6). doi: 10.3201/eid2606.200320. Online ahead of print.

13. Yang Y, Lu Q, Liu M, et al. Epidemiological and clinical features of the 2019 novel coronavirusoutbreak in China.medRxiv preprint doi:https://doi.org/10.1101/02.10.20021675.

14. Qin C, Zhou L, Hu Z,et al. Dysregulation of immune response in patients with COVID-19 in Wuhan, China. Clin Infect Dis. 2020 Mar 12 :ciaa248. doi: 10.1093/cid/ciaa248. Online ahead of print.

15. Wong CK, Lam CW, Wu AK, et al. Plasma inflammatory cytokines and chemokines in severe acute respiratory syndrome.Clin Exp Immunol.2004 ;136(1):95-103.

16. Mahallawi WH, Khabour OF, Zhang Q, et L. MERS-CoV infection in humans is associated with a pro-inflammatory Th1 and Th17 cytokine profile. Cytokine. 2018; 104:8-13.

17. Hodgson K,Morris J,Bridson T,et al.Immunological mechanisms contributing to the double burden of diabetes and intracellular bacterial infections.Immunology. $2015 ; 144(2): 171-85$.

18. Chinese Diabetes Society. Guidelines for the prevention and control of type 2 diabetes in China (2017 Edition). Chinese Journal of Practical Internal Medicine.2018;38(4):292-344

19. Wang F, Nie JY, Wang HZ, Zhao Q, Xiong Y, et al. Characteristics of Peripheral Lymphocyte Subset Alteration in COVID-19 Pneumonia. J Infect Dis. 2020;221(11):1762-1769

20. Wu C, Chen X, Cai Y,et al. Risk Factors Associated With Acute Respiratory Distress Syndrome and Death in Patients With Coronavirus Disease 2019 Pneumonia in Wuhan, China.JAMA Intern Med. 2020 Mar 13:e200994. doi: 10.1001/jamainternmed.2020.0994. Online ahead of print.

21. Yang J, Zheng Y, Gou X,et al.Prevalence of comorbidities in the novel Wuhan coronavirus (COVID-19) infection: a systematic review and metaanalysis.Int J Infect Dis. 2020 Mar 12:S1201-9712(20)30136-3. doi: 10.1016/j.ijid.2020.03.017. Online ahead of print.

22. Yang J,Feng Y,Yuan MY,et al.Plasma glucose levels and diabetes are independent predictors for mortality and morbidity in patients with SARS.Diabet Med. 2006;23(6):623-8.

23. Badawi A, Ryoo SG.Prevalence of Diabetes in the 2009 Influenza A (H1N1) and the Middle East Respiratory Syndrome Coronavirus: A Systematic Review and Meta-Analysis.J Public Health Res. 2016; 5(3):733.

24. Elyahu Y, Hekselman I, Eizenberg-Magar I, Berner O, Strominger I, et al. Aging promotes reorganization of the CD4 T cell landscape toward extreme regulatory and effector phenotypes.Sci Adv. 2019; 5(8):eaaw8330.

25. Muggen AF, de Jong M, Wolvers-Tettero ILM, Kallemeijn MJ, Teodósio C, et al. The presence of CLL-associated stereotypic B cell receptors in the normal BCR repertoire from healthy individuals increases with age.Immun Ageing. 2019; 16:22.

26. Raphael I, Nalawade S, Eagar TN,et al. T cell subsets and their signature cytokines in autoimmune and inflammatory diseases.Cytokine. 2015; 74(1):5-17.

27. Min CK, Cheon S, Ha NY, et al. Comparative and kinetic analysis of viral shedding and immunological responses in MERS patients representing a broad spectrum of disease severity. Sci Rep. 2016; 6:25359.

28. Channappanavar R, Perlman S. Pathogenic human coronavirus infections: causes and consequences of cytokine storm and immunopathology. Semin Immunopathol.2017; 39(5): 529-39

29. Arora S, Dev K, Agarwal B,et al. Macrophages: Their role, activation and polarization in pulmonary diseases. 2018; 223(4-5):383-396.

30. de Candia P, Prattichizzo F, Garavelli S, De Rosa V, Galgani M, et al. Type 2 Diabetes: How Much of an Autoimmune Disease? Front Endocrinol (Lausanne). 2019; 10:451.

31. Boutens L, Stienstra R.Adipose tissue macrophages: going off track during obesity.Diabetologia. 2016; 59(5):879-94.

Page 9/10 
32. Chatzigeorgiou A, Karalis KP, Bornstein SR,et al. Lymphocytes in obesity-related adipose tissue inflammation.Diabetologia.2012;55(10):25832592.

33. Xia C, Rao X, Zhong J.Role of T Lymphocytes in Type 2 Diabetes and Diabetes-Associated Inflammation.J Diabetes Res. 2017; 2017:6494795.

\section{Supplementary Files}

This is a list of supplementary files associated with this preprint. Click to download.

- DataofCOVID19patients.sav.xls 